\title{
Médiévales
}

Langues, Textes, Histoire

\section{Entre visions angéliques et transes chamaniques : le sabbat des sorcières dans le Formicarius de Nider}

Between angelic visions and shamanistic trance: the Witches' Sabbath in the Formicarius of Nider.

\section{Gábor Klaniczay}

\section{OpenEdition}

Journals

Édition électronique

URL : https://journals.openedition.org/medievales/710

DOI : 10.4000/medievales. 710

ISSN : $1777-5892$

Éditeur

Presses universitaires de Vincennes

Édition imprimée

Date de publication : 1 juin 2003

Pagination : 47-72

ISBN : 2-84292-142-9

ISSN : 0751-2708

Référence électronique

Gábor Klaniczay, «Entre visions angéliques et transes chamaniques : le sabbat des sorcières dans le Formicarius de Nider », Médiévales [En ligne], 44 I printemps 2003, mis en ligne le 14 février 2006, consulté le 22 avril 2022. URL : http://journals.openedition.org/medievales/710 ; DOI : https://doi.org/ 10.4000/medievales.710

Ce document a été généré automatiquement le 22 avril 2022

Tous droits réservés 


\title{
Entre visions angéliques et transes chamaniques : le sabbat des sorcières dans le Formicarius de Nider*
}

\author{
Between angelic visions and shamanistic trance: the Witches' Sabbath in the \\ Formicarius of Nider.
}

Gábor Klaniczay

1 Probablement inspiré des controverses du concile de Bâle, achevé aux alentours de 1435, le Formicarius de Johannes Nider (1380-1438) décrit des saints et des hérétiques, des visions, des révélations, des phénomènes de possession et de «simulation, des vertus extraordinaires et des péchés mortels, des opérations miraculeuses, et des œuvres de magiciens et de sorciers $»^{1}$. Un éventail aussi large, qui comprend le bien et le mal dans le même cadre analytique et moral, représente une innovation importante susceptible d'expliquer le succès de ce livre (27 manuscrits, trois éditions en incunables et cinq éditions imprimées²). Pour certains spécialistes, le Formicarius constitue en fait un catalogue d'exempla et nous savons que ce genre relève des règles du folklore autant que des critères du vrai ${ }^{3}$. Nider lui-même nomme souvent ses histoires des exempla, même si ces histoires semblent bien différentes des exempla classiques du XIII et du XIV ${ }^{\mathrm{e}}$ siècles, dont les objectifs pastoraux s'enracinent dans les stéréotypes folkloriques. Dans la tourmente du concile de Bâle, au milieu de cette bourse galopante aux informations, la plus grande partie de la collecte faite par Nider s'apparente davantage à un travail de journalisme impressionniste, bien qu'elle comprenne aussi des exempla de type plus ancien.

2 Les histoires contenues dans le Formicarius ont été l'une des sources principales d'un des manuels les plus importants de démonologie du début de la période de la chasse aux sorcières, le Malleus Maleficarum de Heinrich Krämer (Institoris) et Jakob Sprenger ${ }^{4}$. C'est pourquoi l'historiographie moderne qui s'est intéressée à Nider s'est surtout 
concentrée sur ses descriptions des accusations de sorcellerie au début du $\mathrm{Xv}^{\mathrm{e}}$ siècle en Suisse $^{5}$. On a fait beaucoup moins attention aux passages tout aussi hauts en couleur qui traitent de la religion visionnaire de la fin du Moyen Âge ${ }^{6}$. Dans ce qui suit, je voudrais au contraire essayer de mettre en valeur l'originalité du schéma conceptuel de Nider: la juxtaposition des visions d'origine céleste ou diabolique qui, selon lui, devaient être examinées dans un même cadre.

Une des caractéristiques communes à ces deux manières d'établir une communication avec le surnaturel est le fait qu'elles reposent toutes deux sur l'état de transe, d'extase et de ravissement. L'évaluation ambivalente de la transe est un élément crucial dans la façon dont s'est modifiée la perception de la sainteté visionnaire mystique et celle de la sorcellerie $\mathrm{au} \mathrm{xv}^{\mathrm{e}}$ siècle. Aussi doit-on faire particulièrement attention à la manière dont Nider traite ce sujet dans son ouvrage.

Dans le livre trois du Formicarius sur "falsis et illusoriis visionibus", on trouve une douzaine d'exempla concernant l'extase simulée comme l'un des phénomènes trompeurs associé aux « faux prophètes ». Après quelques anecdotes de bégards suisses qui agissent comme intermédiaires pour dialoguer avec les âmes des défunts, prêchent une «nouvelle règle » ou s'engagent dans les dépravations nocturnes, ${ }^{7}$ Nider raconte l'histoire d'un jeune garçon de treize ans, telle qu'elle lui a été rapportée par l'inquisiteur dominicain, Heinrich Kalteisen. Le garçon fut envoyé à l'école dominicaine de Bois-le-Duc. "Une fois, alors qu'il était entré dans le jardin du couvent, il a vu quelque chose de blanc sur une feuille. Sans prononcer une bénédiction, il l'a arraché et avidement avalé, et ainsi, par manque de souci, il a englouti un diable terrible $»^{8}$. Presque immédiatement, il tomba en transe, son corps immobile ne répondant à aucun stimulus extérieur (rapi eum fratres viderunt et privari omnibus exterioribus sensibus corporis). L'extase du garçon devint dérangeante par la suite. Quand le garçon revint à lui, il commença à parler latin et français couramment (alors qu'il ne connaissait aucun mot de ces deux langues); il citait des passages bibliques par cœur, effectuait des miracles, et avait des visions et des révélations. "Quelques femmes crédules et irréfléchies croyaient reconnaître la présence de l'esprit de Dieu là où le Diable venait d'occuper le terrain $»^{9}$. Les dominicains considéraient tout ceci avec la plus grande suspicion ; ils ne croyaient pas qu'un novice mal dégrossi puisse faire de tels progrès en si peu de temps. À l'aide d'une hostie bénite, ils réussirent à faire voir la tromperie : «en réalité » toute l'affaire prouvait l'œuvre du démon qu'ils réussirent alors à exorciser.

Des états d'extase déroutants de cette nature - comme le Paresseux, l'un des protagonistes du Formicarius, le rappelait à son mentor - survenaient principalement dans les vies des saintes de "grande réputation", mais "la plupart des gens éduqués considèrent que des démonstrations de cette sorte sont de simples simulations ${ }^{10}$. Même si Nider lui-même partage cette réserve, il rappelle à son élève de ne pas juger trop rapidement: "Les effets de l'amour céleste (comme nous l'enseigne le divin Denys) ne sont pas moins puissants que ceux de l'amour humain, ils sont même plus puissants. Nous constatons ceci car le seul fait de penser à l'Aimé est suffisant pour devenir délirant et se mettre à soupirer, gémir, pleurnicher, chanter et pleurer ouvertement $\aleph^{11}$. Nider donne un certain nombre d'exemples pour valider son scepticisme à propos des femmes visionnaires, sur lesquels on reviendra. Il décrit aussi la célèbre anecdote de la vetula dementata, qui affirmait être portée à travers les airs 
lors d'un vol nocturne avec Diane, mais après examen des témoins, son assertion se révélait fausse ${ }^{12}$.

6 Après avoir passé en revue les motifs concernant l'extase ("réelle ou simulée ») des mystiques et des devins populaires, Nider rapporte aussi dans le livre $\mathrm{V}$ de son ouvrage quelques motifs extatiques concernant les sorcières, sur la base des procès verbaux des procès pour sorcellerie menés par le juge Pierre, dans le Simmental dans le voisinage de Berne, entre 1392 et $1406^{13}$. Un autre groupe d'informations provient de l'inquisiteur dominicain d'Autun. Sans détailler ici mon analyse, je voudrais seulement souligner que Nider, tout à l'heure sceptique à l'égard du vol nocturne de la vetula, répète les récits embryonnaires du sabbat des sorcières, c'est-à-dire ceux qui contiennent un certain nombre d'éléments importants qui seront plus tard associés au concept du sabbat, comme le vol extatique nocturne, les fêtes, les orgies, les danses et les copulations de toutes sortes ${ }^{14}$; et il le fait sans beaucoup de réserve. Il reproduit aussi l'histoire des sorciers qui ont fait tomber Pierre du haut de l'escalier ${ }^{15}$. Il n'est pas surprenant que le disciple (Piger) du Formicarius se demande à plusieurs reprises si ces démons agissent en phantasia ou realiter ${ }^{16}$. Nous percevons clairement que Nider luimême se bat dans son explication de l'extase et de la transe avec des alternatives non résolues.

7 Si on étudie les histoires de Nider dans le cadre des théories modernes portant sur la transe et l'extase ${ }^{17}$, on s'aperçoit qu'elles contiennent un certain nombre de motifs qui nous incitent à essayer de confronter les descriptions de transes des visionnaires de la fin du Moyen Âge avec les modèles des " techniques archaïques de l'extase ", à savoir le chamanisme ${ }^{18}$. Il n'est nul besoin de dire que, dans ce qui suit, il n'est en aucune façon impliqué que le phénomène religieux représenté par les mystiques de la fin du Moyen Âge devrait être classé dans une sorte de chamanisme au sens large ; la confrontation permet plutôt d'éclairer certains traits communs afin d'élaborer une typologie historique plus large des types de contact avec le surnaturel.

De ce point de vue, la description la plus instructive fournie par Nider pourrait être celle de ce jeune novice dominicain de Bois-le-Duc. Le garçon a treize ans au moment de sa première transe publique décrite par Nider. C'est environ l'âge auquel un jeune candidat chamane dans le chamanisme de Sibérie est d'abord " appelé » lors d'un rêve à rejoindre la compagnie des chamanes plus âgés pour remplir le rôle qui lui est attribué dans sa destinée ${ }^{19}$. La fonction de la «maladie chamane » initiatique est « l'acquisition du savoir ». Dans le cas du novice dominicain c'est aussi la transe qui donne au jeune garçon sa connaissance du français et du latin, sa perspicacité à lire la Bible, ainsi que le pouvoir de faire des miracles et de recevoir des révélations. Les dominicains interprétèrent ces pouvoirs comme le résultat de la possession du diable. Or, en fait, la possession n'est pas si éloignée du phénomène de la transe chamane qu'on pourrait le penser à première vue. Selon Nider, l'élément déclencheur de la transe est le fait que le garçon sans réfléchir avala "quelque chose» de blanc. Les épisodes mineurs qui suivent comme le ravissement du jeune novice possédé, ses miracles et ses prophéties peuvent être considérés comme des mises en pratique concrètes et routinières de ses capacités acquises durant sa première transe. Ce modèle est connu des anthropologues qui s'occupent du chamanisme dans les sociétés tribales ${ }^{20}$.

9 Néanmoins, avant de voir jusqu'à quel point les catégories du chamanisme peuvent s'appliquer aux autres phénomènes religieux discutés par Nider, nous devons poser rapidement deux autres questions. Peut-on parler de chamanisme, dans une forme 
même fragmentaire, dans l'Europe du haut Moyen Âge ? Et si tel est le cas, peut-on montrer des corrélations d'une part entre le chamanisme et les manifestations d'extase religieuse de cette époque, ou d'autre part, l'émergence à la fin du Moyen Âge des croyances en la sorcellerie?

Concernant la première question, un certain nombre de résultats ont été publiés sur le sujet ces dernières années. Après avoir découvert et analysé les croyances chamanistiques des benandanti du Frioul qui furent jugés comme sorciers aux $\mathrm{xVI}^{\mathrm{e}}$ et $\mathrm{XVII}^{\mathrm{e}}$ siècles $^{21}$, Carlo Ginzburg a publié une autre synthèse complète sur les origines du mythe du sabbat des sorcières ${ }^{22}$. En tenant compte des réactions suscitées par son livre sur les benandanti, il fait une analyse comparée de tout ce qu'il sait des activités de leurs équivalents européens (kresnik ${ }^{23}$, táltos ${ }^{24}$, calusari ${ }^{25}$, donni di fuora ${ }^{26}$, etc.) : leurs voyages spirituels, leurs activités de chamanes, les luttes pour la fertilité et les contacts avec les morts. Bien que les données concrètes pour tous ces cas ne remontent pas plus loin que le début de l'ère moderne, il est peu probable que les villages médiévaux d'Europe n'aient rien su de ceux-ci ou d'autres magiciens bénéfiques. Parallèlement, les recherches menées par Éva Pócs ont découvert un autre domaine important de systèmes de croyances qui a joué un grand rôle dans la formation du concept de sabbat des sorcières, celui des mythologies ambivalentes des fées. Et ces mêmes enquêtes comparatives l'ont aussi conduite à des énoncés nouveaux et déterminants concernant les rapports entre les croyances hongroises dans l'existence des táltos et le chamanisme sibérien. Ces croyances chamaniques ont été en partie "christianisées » et déformées, et ont pu se fondre dans les croyances modernes concernant la sorcellerie ${ }^{27}$. Les vols nocturnes, les éléments extatiques dans les fêtes des sorcières, les métamorphoses animales, les communications avec les morts, constituent autant d'ingrédients empruntés à la mythologie des fées et au "substrat chamanique ", exhumés afin de trouver leur chemin dans le stéréotype démonologique médiéval de l'alliance des sorcières avec le démon. Dans son récent ouvrage intitulé Saintes ou sorcières? Le sort des femmes notoires pendant le Moyen Âge et les Temps modernes, Peter Dinzelbacher reprend cette question: "pourrions-nous prolonger un peu l'interrogation de Ginzburg, et interpréter comme variantes plus récentes du chamanisme non seulement quelques parties de la sorcellerie, mais aussi certains phénomènes mystiques? ${ }^{28}$. Sans réellement en examiner la possibilité, il en rejette rapidement l'idée, tout en remarquant des analogies significatives. À partir de la lecture de Nider, c'est cette hypothèse, celle d'une corrélation entre le chamanisme, les manifestations d'extase religieuse et l'émergence des croyances en la sorcellerie, que nous voudrions reprendre, en examinant successivement les liens du chamanisme avec la sainteté de la fin du Moyen Âge, puis avec le sabbat des sorcières, pour finir par montrer dans quelle mesure la transe peut être considérée comme un point commun entre la sainte et la sorcière, qui constituent un système binaire dont la polarité bascule au $\mathrm{Xv}^{\mathrm{e}}$ siècle.

Culte des saints, chamanisme et « saintes vivantes »

11 Le point de départ de cette corrélation pourrait être le rôle que le possesseur de pouvoirs surnaturels, le personnage du sorcier ou du saint, joue, au moins aux yeux des autres, dans sa communauté. De ce point de vue, on trouve des ressemblances typologiques entre le chamanisme et le culte chrétien des saints. Dans les deux cas, nous avons affaire à un mécanisme de défense contre la malchance et d'appel au surnaturel visant à mettre la communauté sous la protection d'un patron qui vit au contact du surnaturel, ou d'une personne qui a des pouvoirs surnaturels capables de 
protéger la communauté des malheurs extérieurs, et qui opère des miracles. Telle est la nature du culte des saints selon Peter Brown ${ }^{29}$. En s'appuyant sur les catégories de l'école anglo-saxonne d'anthropologie, principalement celles d'Edward EvansPritchard ${ }^{30}$, P. Brown montre que le culte des saints opérateurs de miracles dans les communautés chrétiennes de l'Antiquité a progressivement éclipsé les techniques habituelles de l'époque pour chasser le malheur, c'est-à-dire les formes habituelles d'accusation concernant la magie et la sorcellerie ${ }^{31}$. Avec leurs pouvoirs reçus, leur capacité spectaculaire de chasser les démons, et l'exemple positif de la sainteté de leur vie, le saint comme la sainte ont tout simplement « dérobé le spectacle ». Ils ont gagné le public qui se tournait auparavant vers les experts traditionnels de "magie ", de telle sorte que les gens ont cessé de s'intéresser aux méthodes capables de détourner la mauvaise fortune, qui avaient pour fonction de découvrir et de "nettoyer» la communauté en trouvant des boucs émissaires et en chassant les sorcières ${ }^{32}$.

Un autre aspect montre bien la pertinence du travail de Peter Brown pour notre compréhension du culte des saints dans la dernière partie du Moyen Âge. Durant une grande partie du Moyen Âge, le culte des saints était fondé sur des miracles opérés par les reliques des saints décédés : « le saint homme » charismatique de l'Antiquité tardive auquel Brown fait référence a seulement survécu comme une construction littéraire, le sujet idéalisé des légendes. Mais à partir du xiie siècle s'opère un changement notable. Les personnifications hétérodoxes et orthodoxes de la vita apostolica, tout comme les prêcheurs itinérants d'une orthodoxie impeccable, comme saint Bernard de Clairvaux et saint Norbert, essayèrent tous de réveiller le sentiment de l'autorité surnaturelle du saint homme dans l'esprit populaire ${ }^{33}$, comme en témoignent les miracles publics de saint Bernard opérés à Cologne en 1146-4734. Avec la venue des ordres mendiants au xiiie siècle, vivre une vie de sainteté devient un enjeu réaliste, voire recommandé. Saint François d'Assise était considéré d'ailleurs par ses compagnons comme rien de moins qu'un alter Christus, un « autre Christ ».

13 La nouvelle forme de sanctification bureaucratisée, la procédure de canonisation monopolisée par le Saint Siège ${ }^{35}$, contribua d'un côté à fournir à la sainteté des critères transparents mais diminua de l'autre la possibilité "d'autogérer " ce domaine. Les germes locaux d'un culte ne pouvaient que très rarement se développer en ces circonstances et s'épanouir en sanctifications canonisées. Le statut de la sainteté devint ainsi, paradoxalement, davantage et moins accessible dans le même temps. La conséquence en fut que pendant le $\mathrm{XIV}^{\mathrm{e}}$ siècle et le $\mathrm{XV}^{\mathrm{e}}$ siècle, il y eut de plus en plus de candidats à la sainteté qui se préparaient délibérément pour cette gloire, vivant comme s'ils sortaient directement de La Légende dorée ${ }^{36}$. Et ceci n'était pas seulement et principalement l'aspiration de certaines femmes et certains hommes : les saints étaient entourés par un groupe d'admirateurs proches ou lointains (confesseurs, disciples, moines, frères, nonnes, des cours entières ou des villes) qui les vénéraient comme des saintes, et espéraient d'elles une protection miraculeuse au milieu des calamités sociales, politiques et naturelles du dernier Moyen Âge ${ }^{37}$.

Ces personnes charismatiques étaient de plus en plus fréquemment appelées «les saintes vivantes» (sante vive ${ }^{38}$ ) par la communauté. Beaucoup de ces femmes visionnaires, comme Angèle de Foligno (1248-1309), sainte Claire de Montefalco (1268-1308), sainte Brigitte de Suède (1303-1379) et sainte Catherine de Sienne (1348-1380), "saintes vivantes ", devinrent des actrices essentielles de la scène religieuse à partir du XIII ${ }^{\mathrm{e}}$ siècle ${ }^{39}$. 

pas comme telles. Il raconte par exemple une conversation qu'il a entendue quand il était encore prieur à Nuremberg lors de la réunion des Électeurs allemands et de l'empereur Sigismond en 1428. Le chancelier commença à rendre publiquement hommage à «la mémoire sainte » de Catherine de Sienne qui n'avait pas encore été canonisée et lut à haute voix sa Vie écrite par Raymond de Capoue, remarquant « combien de pécheurs cette vierge avait réussi à convertir en Italie $»^{40}$. En entendant cela, l'ambassadeur du Prince de Savoie commença à relater les miracles opérés en France par la Mère Supérieure des Clarisses, Colette de Corbie (1381-1447), et comment cette domina tantae sanctitatis levait les bras en signe de supplication et convertissait les pécheurs et les incroyants pour que tout le monde puisse voir sa saintetét1. Nider mentionne aussi les deux prêcheurs célèbres de l'époque, saint Vincent Ferrier et saint Bernardin de Sienne, faisant référence à eux comme aux deux nouveaux " prophètes " de Dieu ${ }^{42}$. Le Formicarius fait aussi allusion à des exemples locaux : Adelaïde de Mulberg, vénérée "par toute la ville de Bâle comme un sanctuaire vivant » (vivo sanctuario) ${ }^{43}$; et Buken, la sancta foemina qui vivait en recluse près de la maison des Chevaliers Teutoniques, et au sujet de laquelle Nider raconte une anecdote pour illustrer le fait qu'« il y a ceux qui utilisent leurs dons pour le bien des autres» et qu'il est bon de prêter attention à ce qu'ils disent. Il raconte qu'un homme d'Église appelé Nicolaus avait rejoint les rangs des pèlerins de la région qui recherchaient Buken pour solliciter ses conseils sur des sujets pratiques et spirituels. Buken lui a demandé où il vivait ; en apprenant que c'était près de l'église, elle l'avertit qu'une catastrophe était sur le point de frapper les gens qui habitaient dans cette partie de la ville. Le prêtre - qui lui-même raconta l'histoire à Nider - regretta amèrement de ne pas avoir prêté attention à son avertissement, car peu de temps après, un violent incendie balaya cette partie de la ville, et détruisit sa maison, ainsi que trois cents autres. ${ }^{44}$

À côté de ces «bonnes" mystiques, qu'il a pu personnellement observer, Nider mentionne d'autres cas susceptibles d'appartenir à cette catégorie de saintes vivantes, mais à l'égard desquels il ne peut cacher son scepticisme. Un cas douteux concerne une femme recluse qui vivait dans une ville proche de Radolfzell. Elle était «souvent allongée, prostrée dans un état d'extase, et quand elle revenait à elle, elle décrivait les révélations secrètes qu'elle avait reçues... Un jour, on commença à propager la nouvelle que les cinq blessures du Christ apparaîtraient sur ses mains, ses pieds et son côté un jour donné $»^{45}$. Une foule de curieux se rassembla pour l'occasion; on trouva la recluse allongée sur le sol de sa cellule (velut in rapto, immobilis, fatua), mais les stigmates n'apparurent pas, à la grande consternation de tous ceux qui croyaient aux " délires " et aux révélations de cette femme (deliramentis et ejus revelationibus). Peu de temps après, la simulatrice (ipsa fictrix) fut obligée de passer devant le tribunal ecclésiastique avec l'une de ses disciples; là, elle se rétracta et se repentit de ses péchés.

17 L'histoire la plus connue est celle de Madeleine de Fribourg (Magdalena Beutlerin), la fille de Margarete von Kenzingen (†1428), dominicaine et mystique renommée. Après son entrée dans l'ordre des Clarisses, elle devint célèbre pour ses fréquentes extases (extaticis visionibus ac a sensu alienationibus) ${ }^{46}$. Avant Noël 1430, elle prophétisa sa mort pour l'Épiphanie, et affirma que ses disciples dévoués pourraient assister à ce spectacle et échapper ainsi aux tortures de l'Enfer. Par la suite, elle refusa toute nourriture pendant dix-sept jours, puis, après avoir reçu l'Eucharistie, elle tomba en transe et confirma une nouvelle fois sa prophétie. La nouvelle fut l'objet d'une grande attention, 
et Madeleine fut honorée de la présence de diverses personnalités ecclésiastiques et séculières, des autorités de la ville, ainsi que de quelques sceptiques. Le jour dit, Madeleine arriva à l'église où le public attendait l'événement, « elle mit sa tête sur les genoux d'une autre sœur clarisse, fut immédiatement ravie en une extase réelle ou fictive, et resta ainsi étendue un moment. Comme les spectateurs étaient curieux de savoir si elle était vivante ou morte, le docteur toucha son pouls aux yeux de tous et affirma qu'elle était vivante... $»^{47}$. Même si ses disciples les plus proches continuèrent à croire en elle, sa renommée s'effondra.

La véritable nouveauté de l'époque réside dans le changement effectué par les laïcs chrétiens, qui ne semblaient pas être satisfaits d'avoir accès seulement aux reliques de leurs saints patrons d'autrefois, et souhaitèrent aussi des "saints vivants " dans leur entourage. Cela étant, la scène était préparée pour que la prétendue sainte devienne " un prophète dans son propre pays "- selon l'allusion biblique de Aviad Kleinberg ${ }^{48}$. C'est ici que l'analogie chamanique peut nous être utile. Dans les deux cas, le possesseur bienfaisant de pouvoirs surnaturels vit au sein de la communauté concernée et sert ses intérêts. Le contact avec le surnaturel est accompli sous la forme d'une transe, d'une extase, et peut servir les besoins de la communauté au sens large (par des révélations prophétiques, en prédisant le futur et en prévoyant les catastrophes), ou fournir une solution plus concrète à des problèmes individuels (comme c'est le cas pour les guérisons). Dans les deux cas aussi, le voyage de l'âme au cours d'une extase peut conduire à travers le pays des morts, et exposer la personne en transe à des esprits qui ne sont pas forcément bienveillants, mais aussi au danger des attaques des esprits malfaisants. Enfin, chamanes et saints - ou possédés démoniaques - doivent produire des preuves visibles et physiques de leurs relations avec le monde spirituel pour établir et sauvegarder leur prestige à l'intérieur de la communauté: sueur, pleurs, cris, évanouissements, sexualité intense, mais aussi blessures, bleus et stigmates. Ainsi, aux environ de 1424, une femme visionnaire qui habitait Bourg-en-Bresse en France se vantait d'avoir sauvé de nombreuses vies de la damnation et montrait les cicatrices des brûlures qu'elle avait endurées au cours de ses missions de sauvetage en Enfer ${ }^{49}$. Dans les histoires de Nider, seul le juge Peter porte les marques corporelles d'une aventure surnaturelle, mais elles attestent sa rencontre avec les sorcières, et le sujet est un peu différent. De manière plus significative, les propos de Nider font référence à la stigmatisation qui constitue la manifestation physique la plus originale des mystiques médiévales ${ }^{50}$.

Les stigmates, les signes de l'union physique ultime avec le Christ souffrant, sont considérés avec beaucoup de scepticisme par Nider, comme le montre l'histoire de la recluse contrariée de Radolfzell. La recluse fait une annonce publique de la venue d'un événement mystique, et se met en transe dans l'attente de sa venue - sous l'œil inquisiteur des témoins assemblés. Elle défie le destin et prend le risque d'encourir un ridicule public, tant elle s'efforce de faire reconnaitre son statut surnaturel au sein d'une communauté sceptique. Le risque qu'elle prend a de nombreux points communs avec la disparition physique pendant plusieurs jours de Madeleine de Fribourg pendant son "extase ", et son invitation adressée à des spectateurs pour qu'ils assistent à sa propre mort qu'elle-même avait annoncée. Nous assistons au même type de motivation dans le cas de la vetula qui s'enduisait le corps avec de l'onguent de sorcière devant les yeux incrédules des dominicains, pour donner du crédit à son témoignage concernant ses vols nocturnes avec Diane. Plus les gens doutaient des choses spirituelles, plus le public religieux $\mathrm{du} \mathrm{xv}^{\mathrm{e}}$ siècle demandait des preuves visibles et publiquement 
vérifiables des miracles et du surnaturel, et plus les visionnaires essayaient, dans une certaine mesure, de satisfaire cette nouvelle demande. C'est pourquoi les stigmates, les signes visibles et extérieurs de l'union physique avec le Christ souffrant, en vinrent à être considérés comme les signes ultimes de l'union mystique.

Cette demande d'authentification des expériences mystiques par des preuves visibles le désir de cette "identification la plus sanglante» (allerblütigiste glicheit) comme la béguine $\mathrm{du} \mathrm{xIV}^{\mathrm{e}}$ siècle, Elsbeth von Oye, appelait dans son autobiographie les châtiments corporels qu'elle s'infligeait ${ }^{51}$ - entrait en contradiction quand elle faisait état des notions traditionnelles de la nature de la transe. En effet, la transe, qui est le signe extérieur le plus significatif de l'extase dans le chamanisme comme dans le christianisme, peut être décrite ainsi : l'âme d'une personne en transe est séparée du corps (ecstasim id est mentis excessum, suivant l'expression usuelle à partir du XII ${ }^{\mathrm{e}}$ siècle ; Nider utilise souvent la même expression ${ }^{52}$ ) ; l'âme est transportée (rapta) dans une sphère plus haute, où elle entre en contact direct avec le monde surnaturel. Le corps inconscient - habituellement inerte, mais qui peut être secoué de convulsions comme s'il était possédé - est insensible à toutes les stimulations physiques extérieures ; quant aux expériences visionnaires pendant l'extase, c'est un «secret » à propos duquel la communauté n'a d'autre information que son compte-rendu postérieur.

21 Le problème est que tout le monde peut simuler l'immobilité ou les convulsions de l'état de transe classique (comme Nider le répète); le " public » religieux du début du $\mathrm{xv}^{\mathrm{e}}$ siècle désirait des preuves plus concrètes. Les gens voulaient être les témoins visuels des apparitions spontanées des stigmates sur les mains et les pieds de la nonne zélée qui était allongée inerte dans sa cellule. Ils voulaient que le docteur prenne le pouls de Madeleine, et qu'il déclare si sa vie déclinait au moment où elle l'avait prophétisé. Avec leurs propres yeux ils voulaient voir la vieille femme s'en aller chevaucher avec Diane.

Dans le cadre du système de croyances chamane traditionnel, personne ne se serait moqué de la vieille « sorcière »- qui, selon Nider, était régulièrement dans un état de transe - quand elle prétendait que "seule » son âme était partie. Le climat troublé du débat religieux de la première décennie $\mathrm{du} \mathrm{xv}^{\mathrm{e}}$ siècle exigeait quelque chose de plus : la légitimation physique de ces expériences surnaturelles. On pourrait penser que tout ceci est à l'origine du processus qui conduira au triomphe éventuel du rationalisme. En fait, ce fut le contraire qui eut lieu. Je suis convaincu que c'est précisément cette attitude critique excessive qui a conduit durant ces décennies au rejet du dogme du premier Moyen Âge formulé dans le canon Episcopi et à l'idée que le vol physique, réel doit être considéré comme impossible. C'est pour arriver à une preuve suffisante pour vaincre cet esprit critique que les premiers tribunaux modernes organisèrent une pression sur les accusés d'une telle ampleur que ces derniers étaient prêts à tout confesser sous la torture. La " preuve » ainsi obtenue attisa considérablement le climat d'anxiété qui se transforma en hystérie au moment où eut lieu la chasse aux sorcières de l'époque moderne.

Nider lui-même est à la limite des deux traditions. Sa raison l'incite à représenter le point de vue sceptique, rationnel ; mais dans le Formicarius, il ne cesse de confesser ses incertitudes et suspend sa faculté critique. En résumé, l'ouvrage en son entier est un pas concret pour rapprocher l'expérience mystique et les visions démoniaques, la sainteté et la sorcellerie selon un nouveau type de dénominateur commun. 
24 Et Nider n'est pas le seul à penser ainsi : ses réflexions s'insèrent dans la discussion contemporaine sur le discernement des esprits (discretio spirituum) ) $^{53}$, à partir de Henri de Friemar ${ }^{54}$, Henri de Langenstein ${ }^{55}$, Jean Gerson ${ }^{56}$, jusqu'aux confrères observants de Johannes Nider: Johannes de Mulberg (qui était le frère d'Adelaïde de Mulberg, la sainte femme de Bâle que Nider mentionne ${ }^{57}$ ), et Eberhard Mardach ${ }^{58}$. Dans le contexte aussi de la «naissance du soupçon» concernant l'authenticité des phénomènes du mysticisme féminin ${ }^{59}$, les frontières entre les apparitions divines et diaboliques devenaient de plus en plus incertaines au point de disparaître complètement dans des cas célèbres comme celui de Jeanne d'Arc, vivement discuté lors du concile de Bâle, et décrit par Nider dans le livre $\mathrm{V}$ de son ouvrage. Dans ce texte, il raconte qu'elle " déclara qu'elle avait avec elle un ange de Dieu qui, au jugement des plus savants, fut estimé être un esprit malin d'après nombre de conjectures et preuves. Cet esprit fit d'elle une sorte de magicienne et ils permirent qu'elle fût livrée au feu par la justice publique... $»^{60}$.

25 Cette facilité pour passer de la sainte à la sorcière et vice versa, illustrée par Nider, nous conduit au problème central de notre réflexion: quels sont les rapports entre d'une part l'ambiguïté envers le mysticisme et le prophétisme féminins, et d'autre part les premières persécutions contre les sorcières, qui survinrent précisément au moment $\mathrm{du}$ concile de Bâle, dans les vallées suisses et alpines, au cours du premier quart $\mathrm{du}_{\mathrm{xv}} \mathrm{e}^{\mathrm{e}}$ siècle? Avant de se prononcer sur ce point, il nous faut tout d'abord rappeler le statut $\mathrm{du}$ sabbat des sorcières au $\mathrm{xv}^{\mathrm{e}}$ siècle, puis $\mathrm{y}$ chercher les liens avec les phénomènes liés à la transe et au chamanisme.

La nouvelle mythologie du sabbat des sorcières

Il existe un consensus dans la recherche historique selon lequel les accusations pour maleficium éparpillées durant le Moyen Âge se sont transformées en accusations de sorcellerie massives et épidémiques à partir du moment où, dans le premier quart du $\mathrm{Xv}^{\mathrm{e}}$ siècle, les notions de la sorcellerie traditionnelle s'amalgamèrent avec la mythologie plus récemment inventée du sabbat diabolique des sorcières ${ }^{61}$. Joseph Hansen a donné la définition suivante de cette nouvelle forme de sorcellerie : « ce sont principalement des femmes, qui passent un pacte avec le démon pour détruire l'humanité avec son aide, elles s'organisent en sectes hérétiques, participent régulièrement au sabbat des sorcières présidé par le démon pendant la nuit, elles se rendent aux réunions en volant dans les airs grâce à l'aide du démon, et elles commettent des actes sexuels infâmes avec lui $»^{62}$. Depuis que la recherche historique reconnaît l'importance de cette mutation du début du $\mathrm{xv}^{\mathrm{e}}$ siècle, il y a des débats constants sur l'importance relative des facteurs divers qui ont contribué à ce changement.

27 L'explication la plus largement acceptée est que les sorcières auraient hérité des principaux éléments du sabbat à partir des stéréotypes inquisitoriaux formulés lors de la persécution des mouvements hérétiques médiévaux, et plus tard des Chevaliers du Temple, accusés eux aussi de messes noires infâmes et de pactes avec le diable ${ }^{63}$. De même, la relation des Vaudois avec les sorcières semble ressortir du fait que le mot vauderie devint au $\mathrm{xv}^{\mathrm{e}}$ siècle l'un des noms les plus fréquents pour désigner la sorcellerie diabolique ${ }^{64}$. Élargissant encore l'enquête, on a récemment proposé comme sources possibles de la sorcellerie diabolique les stéréotypes du XIV siècle formulés lors des persécutions d'autres boucs-émissaires, tels que les juifs et les lépreux ${ }^{65}$. De plus, l'analyse détaillée de plusieurs centaines de procès de sorcières du début $d u \mathrm{xv}^{\mathrm{e}}$ siècle 
en Suisse, dans le Dauphiné et en Savoie, et leur confrontation avec d'autres procès visant dans la même région les Vaudois - qui se retirèrent dans les Alpes à la fin du Moyen Âge - ou encore à d'autres hérétiques ont confirmé les effets des procédures inquisitoriales sur l'émergence du nouveau stéréotype que constituait le sabbat des sorcières ${ }^{66}$.

Mais deux autres hypothèses peuvent également être évoquées: d'abord, le fait que dans les procès, qui commencèrent à se multiplier à la fin du XIV ${ }^{e}$ siècle, les accusations traditionnelles pour maleficia étaient de plus en plus souvent interprétées suivant le lexique de l'invocation démoniaque et de la "magie noire ", telle qu'elle apparaissait dans le genre désormais prolifique des livres magiques ${ }^{67}$; la sorcellerie proviendrait alors d'une évolution du statut des rituels magiques. Enfin, la sorcellerie pourrait puiser ses origines dans les diverses mythologies populaires, ainsi que le suggère l'analyse des croyances chamaniques ou des croyances dans les fées, hypothèse dont nous avons déjà eu l'occasion de parler ici.

Nous trouvons chez Nider l'une des premières descriptions des réunions secrètes de la secte des sorcières adorant le démon, sacrilèges et dévoreuses d'enfants. On pourrait s'attendre à ce que le premier groupe d'explications sur l'origine du sabbat des sorcières, celui qui met en avant les stéréotypes inquisitoriaux anti-hérétiques, trouve un soutien considérable dans ces descriptions. En effet, les scènes d'orgies extatiques et de fêtes de la secte hérétique libertine où l'on déflore une virgo de soixante-six ans que Nider ne rattache certes pas à la secte des sorcières décrite dans un autre chapitre de son livre - fournissent cependant autant d'aperçus intéressants concernant la création de la nouvelle image du sabbat des sorcières, à l'instar des récits du juge Pierre, ou de l'inquisiteur dominicain d'Autun ${ }^{68}$. Néanmoins, il est difficile de comprendre pourquoi Nider, qui fournit un récit prolixe sur les Hussites ${ }^{69}$, les Adamites et les frères du Libre Esprit, ne consacre pas une seule ligne aux Vaudois, mise à part une référence sans consistance ${ }^{70}$. Cela est d'autant plus curieux qu'à Fribourg, en 1430, au moment même où Nider suivait avec beaucoup d'attention les extases spectaculaires de Madeleine Beutlerin, se déroulaient des procès contre les Vaudois, sous l'égide de l'inquisiteur Ulric de Torrenté, qui devint ensuite célèbre pour ses persécutions implacables de sorcières aux alentours de 1438 dans la région de Vevey et de Neuchâtel ${ }^{71}$.

Si l'on regarde de près les explications de Nider en fonction de la deuxième hypothèse, c'est-à-dire celle d'un rituel magique qui conduirait à la nouvelle mythologie diabolique du sabbat, on trouve aussi des indications utiles. La figure très détaillée du sorcier Scaedeli, que Nider nomme in maleficii magistrum, soumis aussi à la torture, ne « confesse » pas les histoires récemment inventées de la secte des sorcières, mais plutôt les histoires traditionnelles du sorcier solitaire qui opère grâce à la " magie noire » : les lézards enterrés sous le seuil de la porte causant la stérilité, l'évocation du prince des démons par des incantations, un poulet noir dont le cou est tranché au croisement d'une route, et qui est par la suite lancé en l'air ${ }^{72}$. Nider mentionne un autre magicien, un moine étrange du couvent des Écossais de Vienne, qui évoque le démon avec l'aide de libros daemonum de Necromantia, et qui, plus tard, se repent de ce péché horrible ${ }^{73}$. L'auteur décrit également les différentes recettes de la magie amoureuse ${ }^{74}$, et même des essais concrets ${ }^{75}$.

31 Pour nourrir la troisième hypothèse sur l'origine de la nouvelle mythologie de la sorcellerie, à savoir l'impact des motifs traditionnels des croyances populaires, on 
découvre dans le livre de Nider un épisode qui propose une description du fonctionnement des opposants traditionnels aux sorcières dans le cadre du village, les devins, ainsi que de leurs ruses populaires que Nider nomme, de manière caractéristique, des maleficia. Ces opposants voient dans le plomb fondu, et essaient de faire souffrir la prétendue sorcière par des moyens symboliques. Nider, néanmoins, ne recommande pas cette manière de se confronter au mal causé par les sorcières : "on devrait plutôt mourir que se tourner vers de telles pratiques ", ou plutôt on devrait se tourner vers les «moyens licites» offerts par la médiation des saints ${ }^{76}$. On peut aussi découvrir la sorcellerie traditionnelle destinée à procurer ou réduire la fertilité dans les récits de Scaedeli; celui-ci était capable de transférer un tiers de la moisson de n'importe qui dans son propre territoire, de provoquer la grêle, la foudre et le vent ravageur et de causer la stérilité chez les animaux et chez les hommes ${ }^{77}$. En somme, on peut considérer que Nider ne donne pas de preuves décisives pour l'une ou l'autre des explications rendant compte de l'origine du sabbat des sorcières.

Grâce aux recherches récentes, on dispose maintenant d'un large faisceau de nouvelles sources à propos des persécutions pour sorcellerie au début $\mathrm{du} \mathrm{xv}^{\mathrm{e}}$ siècle dans les régions alpines: le traité d'un juge séculier Claude Tholosan intitulé Ut magorum et maleficiorum errores manifesti ignorantibus fiant, daté des alentours de $1436^{78}$ et fondé sur plusieurs centaines de procès de sorcières dans la région de Briançon ${ }^{79}$; le traité Errores Gazariorum, auquel l'édition et les analyses nouvelles assignent un rôle encore plus fondamental que celui qu'on lui prêtait ${ }^{80}$, et le récit composé par le chroniqueur lucernois Hans Fründ au sujet des chasses aux sorcières en Valais entre 1428 et $1430^{81}$. À partir de ces documents, qui ont récemment été confrontés aux énoncés de Nider par diverses analyses ${ }^{82}$, nous obtenons une image plus détaillée des caractéristiques de cette " toute récente secte » des sorcières, et nous comprenons mieux comment s'est formée la mythologie du sabbat des sorcières telle que nous la révèlent les siècles postérieurs. Je voudrais ici seulement évoquer deux points spécifiques: les liens éventuels avec le chamanisme et l'image de "la sorcière au village ${ }^{83}$ dans ces nouvelles descriptions.

Le sabbat, l'image inverse du chamanisme au village?

Pour les parallèles avec le chamanisme, Carlo Ginzburg, dans son analyse récente de ces mêmes sources, considérait la description détaillée du vol magique des sorcières au sabbat comme l'élément le plus significatif. Claude Tholosan d'une part met l'accent sur le fait que les sorcières qui disent se déplacer physiquement (corporaliter) aux assemblées (synagoga) sont une "illusion » suggérée par le démon dans leur sommeil ; d'autre part, il consigne pourtant chaque bribe d'information sur le sujet dans son texte. Il explique que les sorcières vont à leur conclave sur leur bâton magique graissé avec l'onguent fabriqué avec des enfants; parfois, elles chevauchent un balai ou le dos d'animaux sauvages ${ }^{84}$. Mais il me semble acceptable de penser que le voyage de l'âme, comme motif du vol extatique des sorcières, la notion d'onguent des sorcières, et la métamorphose de l'âme quittant le corps sous la forme d'un animal, puissent être interprétées avec Carlo Ginzburg comme le "substrat chamanique " de la culture européenne. À partir du moment où on a parlé pour la première fois du vol d'une sorcière pour l'assemblée, le vol de l'âme devint un leitmotiv de toute la mythologie des sorcières. Nous pouvons détecter des influences moins directes dans l'idée d'un démon principal donnant la cadence du quadrille des sorcières en frappant son tambour ${ }^{85}$, et dans le fait que des démons prennent la forme d'animaux comme l'ours et le bélier. Dans la même veine, nous trouvons Hans Fründ - et les procès des sorcières qu'il 
décrit - qui relie la notion de loups-garous à celle de sorcières, et accuse les sorcières de se transformer en loups et de piller les troupeaux de moutons et de chèvres ${ }^{86}$.

Bien que la croyance aux loups-garous soit sans aucun doute d'origine chamanique ${ }^{87}$, cette accusation est surtout en rapport avec un autre facteur de développement à grande échelle des witch panics, à savoir le fait d'identifier la sorcière comme la cause des déboires agricoles de la communauté. Cette partie des Errores Gazariorum a reçu moins d'attention que les récits morbides du sabbat des sorcières; pourtant, le texte donne des détails sur ce qui était probablement un lieu commun au Moyen Âge. Si « les gens meurent dans certaines villes et les villages, et que dans d'autres régions voisines règne le plus grande insalubrité de l'air $»^{88}$, ce sont les sorcières qui sont responsables, car elles répandent dans l'air depuis le sommet des montagnes leur poudre faite à partir d'animaux vénéneux. "Par mauvais temps ils se sont réunis en grand nombre dans les montagnes, à la demande du diable, pour briser de la glace... [ils] charient la glace dans l'air pendant une tempête, avec l'aide du diable, en se servant aussi de leur bâton, pour détruire les terres fertiles de leurs ennemis et de tous les voisins ${ }^{89}$. Les préoccupations quotidiennes sont présumées concerner aussi les sorcières. Selon l'auteur du traité anonyme, le démon attire plus vraisemblablement ceux «qui ne peuvent vivre pacifiquement sans s'adjoindre beaucoup d'ennemis ». Un autre groupe de candidats est constitué de ceux qui sont devenus pauvres à la suite d'une vie de débauche, mais qui ne veulent pas renoncer à leur style de vie dissolue : ceux-là, le diable «les conduit dans les maisons de puissants dignitaires, nobles bourgeois et autres, dans les maisons desquels il sait que se trouvent les nourritures et le vin qui vont satisfaire leur volonté et leur désir... Ils y restent jusqu'au milieu de la nuit environ... après avoir suffisamment mangé et bu, chacun retourne à ses affaires $»^{90}$. Cette razzia sur les provisions, variante du sabbat des sorcières, et qui est aussi fréquente dans les descriptions provenant des villageois hongrois ${ }^{91}$, tient une place importante dans le récit de Hans Fründ. Il explique comment les sorcières utilisent " des matières viles" (bos materye) pour remplacer le vin volé dans les caves et donne un récit détaillé de ce qui incite les gens à la sorcellerie. Le malin leur promet la richesse, ainsi que la puissance et la possibilité de se venger de ceux qui leur ont fait du tort: «il convainc ces mêmes personnes en s'appuyant sur l'orgueil, l'avarice, la jalousie, la haine et l'hostilité qu'un homme peut ressentir à l'encontre de son prochain $»^{92}$. Les documents des procès du Dauphiné évoquent nombre de ces accusations : ainsi celle d'empoisonner le puits d'un voisin ${ }^{93}$.

Remarquons que ce ne sont pas des contes fantastiques racontés sous la torture. Cela reflète le raisonnement du chasseur de sorcière traditionnel, ou plutôt la tension moralisatrice de ce raisonnement: la tendance à projeter sur ces femmes l'image de l'ennemi collectif qui contrevient à la cohésion sociale et économique de la communauté. Analysant le récit de Nider, tout particulièrement les accusations lancées à l'encontre de Scaedeli, Arno Borst a attiré l'attention sur les tensions sociales dissimulées derrière les chasses aux sorcières ${ }^{94}$, selon une approche qui relève de "la sociologie des accusations $»^{95}$ en vogue parmi les chercheurs anglo-saxons.

$\mathrm{Vu}$ sous ce jour, le fait que, pendant la chasse aux sorcières des années 1430, les sorcières étaient les boucs émissaires des problèmes liés à la fertilité de la communauté - tant personnelle qu'agricole - qui sont traduits en des termes plus dangereux et effrayants que jamais, doit sembler aussi significatif que la mise en place, à la même époque, du sabbat comme fin ultime de la mythologie des sorcières. Le 
nouveau lexique décrit une sorcière qui n'est plus motivée par les intérêts personnels et familiers de la jeteuse de sorts traditionnelle et du voleur de lait de vache; la nouvelle sorcière appartient à une secte organisée, qui saccage et pille avec l'aide du démon, qui possède des poisons efficaces avec lesquels elle détruit tout. Ces potions ne sont pas des philtres d'amour pour allumer le désir ou rendre impuissant; ces sorcières tuent des petits enfants, les leurs et ceux des autres (dans le ventre des mères ou dans le lit des parents) ; les pratiques dans lesquelles elles s'engagent ne sont pas seulement immorales, mais perverses : ce sont des orgies impitoyables, au cours desquelles les sorcières deviennent elles-mêmes les concubines du diable.

La transe, entre sainteté et diablerie

37 Je me suis apparemment éloigné de mon sujet, qui est de savoir comment Johannes Nider considérait l'état de transe dans le Formicarius, et quel effet ses explications eurent sur la pratique de la chasse aux sorcières qui s'en suivit. Mais j'ai délibérément choisi de faire ces digressions : ce sont les théories sociologiques et anthropologiques de la sorcellerie qui nous montreront les corrélations entre ce que dit Nider des visionnaires de la fin du Moyen Âge, l'état de transe et les sorcières.

Si nous nous rappelons ce que nous avons dit plus haut au sujet de l'analogie entre les fonctions religieuses des saints extatiques du haut Moyen Âge et les fonctions communautaires des chamanes devins et guérisseurs comme d'autres artisans de la "magie blanche ", nous verrons comment l'interprétation médiévale de la sorcière en tant qu'associée du diable trouve sa place dans ce tableau. Le rôle négatif révoltant donné à la sorcière joue une rôle non négligeable dans la réévaluation religieuse et culturelle qui donna aux « saintes vivantes » leur prestige grandissant : tout cela faisait partie de la croyance de plus en plus grande dans l'existence de médiateurs humains, personnifiant des pouvoirs surnaturels. Il s'agit donc d'une corrélation typologique qui eut des conséquences étendues.

Cela eut un impact d'une part sur les mythologies qui entourèrent de plus en plus les saintes visionnaires, et d'autre part, les sorcières adoratrices du démon. Des analogies étonnantes, qui allaient dans les deux sens, se développèrent en effet entre ces deux catégories au cours $\mathrm{du} \mathrm{Xv}^{\mathrm{e}}$ et $\mathrm{XVI}^{\mathrm{e}}$ siècles. Il y eut un effet de miroir entre les deux pôles du panthéon surnaturel, du fait qu'ils étaient, d'une certaine manière, les deux côtés d'une même pièce. Mais l'effet de miroir fonctionnait aussi à cause de leur ambivalence qui bientôt brouilla la distinction entre les deux, comme le montrent de récentes recherches. La projection de la vision angélique sur la vision diabolique, et vice-versa, et l'enrichissement des deux motifs qui en découla, commencent seulement à être étudiés ${ }^{96}$.

Le Formicarius de Nider nous permet de penser que l'une des sources des analogies pourrait tenir sans doute dans le fait que les gens qui avaient affaire aux visions des saints, à leurs miracles et à leurs révélations étaient les mêmes qui s'occupaient de la mythologie du sabbat des sorcières. Au Xve siècle, l'hagiographie et la démonologie croissaient sur le même arbre. Les théologiens du Moyen Âge tardif nous font clairement voir que leurs intérêts se rapportaient à ces deux pôles : outre Nider, on peut mentionner son important précurseur, Jean Gerson, qui, à côté de ses travaux se rapportant à la théologie mystique et de ses diatribes à l'encontre des femmes visionnaires, consacra plusieurs traités au problème de la superstition et de la sorcellerie (De diversis diaboli tentationibus, Contra superstiosam diem observationem, Contra errores magicae) ${ }^{97}$. 
41 Tandis qu'à l'époque médiévale, la sainte a été l'actrice principale de la scène religieuse de l'Europe, les ambiguïtés de la première moitié $d u x^{e}$ siècle conduisirent une partie de la Chrétienté à répudier complètement ce culte à l'époque moderne. D'autre part, les sorcières gagnèrent en importance jusqu'au XVII siècle ; la sorcière fut, sans doute, la «star» du panthéon surnaturel de l'Europe au début de l'époque moderne. Rappelons ce qui a été dit au sujet des similarités fonctionnelles et des différences entre le culte des saints et la chasse aux sorcières en tant qu'ils étaient des réponses au malheur et des moyens pour prévenir le malheur. Nous pourrions dire que, tandis que durant le Moyen Âge la croyance dans la capacité du saint à protéger la communauté contre le malheur semblait la solution la plus attirante (en témoigne la multiplication des cultes locaux et des «saintes vivantes»), les Européens du début de l'Époque moderne préférent essayer de dénicher en leur sein ceux qui, pour leur gain personnel et avec l'aide du diable, leur font du mal, et essaient de rétablir l'équilibre en faisant brûler le bouc émissaire.

Nous sommes ici face à deux systèmes très différents mais en aucune façon exclusifs l'un de l'autre. On sait que les procès de sorcières n'étaient pas inconnus au Moyen Âge ${ }^{98}$, et plus d'un saint avait la réputation d'avoir protégé les croyants de sorts jetés par des magiciens et des sorcières ${ }^{99}$. On sait aussi que le culte des saints se poursuivit jusqu'à l'Époque moderne et devint plus vigoureux et que, dans les pays catholiques, les gens continuèrent à se tourner vers les saints et leurs reliques pour contrer l'œuvre des sorcières ${ }^{100}$. L'époque était très conflictuelle quand, à la fin du Moyen Âge, au moment où la croyance en la sorcellerie était en progression, une "sainte vivante » fut appelée pour s'occuper du nouveau type de sorcière. Dans le procès de canonisation de sainte Brigitte de Suède, on peut lire que ses prières libérèrent un prêtre au bord de la folie d'un charme d'amour - et de la sorcière elle-même qui se suicida ${ }^{101}$.

Les deux stratégies face au malheur coexistaient dans les villages à la fin du Moyen Âge et à l'Époque moderne. Parmi ceux qui traditionnellement enlèvent les charmes des sorcières, on trouve des figures de magiciens de type chamane comme le benandante et le táltos, patrons de la communauté dotés de pouvoirs surnaturels, qui avaient recours aux mêmes mécanismes que les saints pour détourner le malheur ${ }^{102}$. Mais quand ils furent amenés à prendre part à la lutte contre la sorcellerie, quand on attendit d'eux leur aide dans l'entreprise de démasquage des traitres à l'intérieur de la communauté, et non plus des esprits démoniaques lointains, quand ils commencèrent eux-mêmes à se joindre à ceux qui accusaient les sorcières, quelque chose changea dans leur autorité surnaturelle. Bientôt se retourna contre eux l'accusation : " celui qui peut guérir, peut ensorceler aussi ».

La boucle est bouclée. Si nous acceptons mon idée que les « saints vivants » de la fin du Moyen Âge furent les « héritiers » des fonctions chamaniques de la magie blanche, et si nous nous souvenons aussi que leur apogée eut lieu au moment où la peur des sorcières démoniaques augmentait, alors il devient évident que les visionnaires de la fin du Moyen Âge ne pouvaient qu'être accueillies de manière équivoque. Si le même processus de réévaluation amena l'émergence des «saints vivants " et des sorcières, ceci n'était vrai qu'en ce qui concerne leur crédibilité comme opérateurs surnaturels. La sainte et la sorcière évoluaient dans deux systèmes de croyance diamétralement opposés. Celui de la sainte, en dépit de ces derniers moments de gloire, était au bord de la désintégration, tandis que celui des sorcières était en pleine mutation et en plein essor. Et de plus en plus souvent, les saintes vivantes eurent le sort de Jeanne d'Arc: 
elles furent déplacées d'un système dans l'autre. Pour l'une, c'était miracle, pour l'autre, sorcellerie.

Le Formicarius oscille de manière incertaine sur les bords de cette mutation. Bien qu'il soit sceptique vis-à-vis des " visions fausses, simulées ", Nider fait rarement mention de leurs origines démoniaques et se tourne avec vénération vers les "vraies " visions et les «vraies saintes vivantes ». Il se moque de ses contemporains qui croient aux vols des sorcières, mais croit qu'elles tuent les enfants. Il n'écarte pas la possibilité que le Bénédictin de Vienne qui avait pratiqué la magie noire soit pardonné comme Théophile. Et il ne fait pas le rapport entre l'extase orgiaque des sectes hérétiques et les orgies des sorcières, que ses proches successeurs feront. Au fond, Nider n'est pas capable de créer de l'ordre sur la scène religieuse chaotique et conflictuelle du Moyen Âge - mais ses efforts annoncent déjà la synthèse que constituera deux générations plus tard le Malleus Maleficarum.

\section{ANNEXES}

*. Une version beaucoup plus longue de cette analyse, tirée du premier chapitre d'un ouvrage à paraître sur La sainteté et la sorcellerie, doit être publiée dans le volume collectif dédié au Formicarius de Johannes Nider par l'ENS Fontenay/Saint-Cloud, dirigé par Nicole Jacques-Chaquin et Jean Céard. Je les remercie de me permettre d'en donner ici une version abrégée par les soins d'Étienne Anheim, à partir de la traduction française de Sophie Houdard.

\section{NOTES}

*.Une version beaucoup plus longue de cette analyse, tirée du premier chapitre d'un ouvrage à paraître sur La sainteté et la sorcellerie, doit être publiée dans le volume collectif dédié au Formicarius de Johannes Nider par l'ENS Fontenay/Saint-Cloud, dirigé par Nicole Jacques-Chaquin et Jean Céard. Je les remercie de me permettre d'en donner ici une version abrégée par les soins d'Étienne Anheim, à partir de la traduction française de Sophie Houdard.

1. K. SCHIELER, Magister Johannes Nider aus dem Orden der Prediger-Brüder. Ein Beitrag zur Kirchengeschichte des fünfzehnten Jahrhunderts, Mayence, 1885 ; E. HILLENBRAND, "Johannes Nider », dans Die deutsche Literatur des Mittelalters. Verfasserlexikon, vol. VI, p. 971-978 ; B. GALBRETH, « Nider and the Exemplum. A Study of the Formicarius », Fabula, Zeitschrift für Erzählforschung, 6, 1963, p. 55-72. Pour l'intérêt récent autour de ce texte, voir M. Brand, Studien zu Johannes Niders deutschen Schriften, Rome, 1998 ; l'édition partielle et le commentaire du Formicarius par Catherine Chène, dans L'Imaginaire du sabbat. Édition critique des textes les plus anciens (1430 ca-1440 ca), réunis par M. Ostorero, A. Paravicini Bagliani, K. Utz Tremp, en collaboration avec C. Chène, Lausanne, 1999, p. 99-265 ; W. Tschacher, Der Formicarius des Johannes Nider von 1437. Studien zu den 
Anfängen der europäischen Hexenverfolgungen im Spätmittelalter, Aix-la-Chapelle, 2000 ; M. D. BAILEY, Battling Demons. Witchcraft Heresy, and Reform in the Late Middle Ages, University Park, Pennsylvania, 2002.

2. T. Kaeppeli, Scriptores Ordinis Praedicatorum Medii Aevi, Rome, 1975, vol. 2, p. 500-515 ; voir L'Imaginaire du sabbat, op. cit., p. 108-120 ; W. Tschacher, op. cit., p. 83-117 ; réimpression de l'incunable de 1480 : Johannes Nider, Formicarius, dir. H. Biedermann, Graz, 1971. J'ai eu accès à l'édition de 1692, Helmstedt, publiée avec le titre altéré De visionibus ac revelationibus; les pages citées renvoient à cette édition.

3. Voir C. Brémond, J. Le Goff et J.-C. Schmitt, L'Exemplum, Turnhout, 1982 (Typologie des sources du Moyen Âge occidental, 40).

4. G. JeROUSCHEK (éd.), Malleus maleficarum 1487 von Heinrich Kramer (Institoris). Faksimile der Handschrift von 1491 aus dem Staatsarchiv Nürnberg, Nr. D. 1521, Hildesheim-Zurich-New York, 1992 ; sur son impact, voir J. HANSEN, Quellen und Untersuchungen zur Geschichte des Hexenwahns und der Hexenverfolgung im Mittelalter, Bonn, 1901, p. 360-410 ; W. STEPHENS, Demon Lovers. Witchcraft, Sex and the Crisis of Belief, Chicago, 2002.

5. J. HANSEN, op. cit., p. 88-99 ; A. BORST, « Anfänge des Hexenwahns in den Alpen », dans ID., Barbaren, Ketzer und Artisten. Welten des Mittelalters, Munich, 1988, p. 262-286 ;

C. Ginzburg, Le Sabbat des sorcières, Paris, 1992 ; M. Bailey, « The Medieval Concept of the Witches' Sabbath ", Exemplaria, 8, 1996, p. 419-439; les passages édités par Catherine Chène dans L'Imaginaire du sabbat sont également ceux qui se rapportent à ce thème. 6. W. WILLIAMS-KRAPP, " "Dise ding sint dennoch nit ware zeichen der heiligkeit". Zur Bewertung mystischer Erfahrungen im 15. Jahrhundert », Zeitschrift für Literaturwissenschaft und Linguistik, 80, 1990, p. 61-71 ; P. DINZELBACHER, Heilige oder Hexen? Schicksale auffälliger Frauen in Mittelalter und Frühneuzeit, Zurich, 1995, p. 25, 66, 89-93, 96, 114, 252-256.

7. Cf. Formicarius, III/1, p. 288 ; III/2, p. 303 ; III/5, p. 338 ; III/7, p. 350-351; J.-C. Schmitt, La Mort d'une hérésie : l'Église et les clercs face aux béguines et aux bégards du Rhin supérieur du XIV au XV $V^{e}$ s., Paris, 1978. Pour les Béguines en Suisse, voir Die Beginen und Begarden in der Schweiz, H. Achermann, C. Sommer-Ramer et alii (éd.), Bâle - Francfortsur-le-Main, 1995 (Helvetia sacra ; Abt. 9, vol. 2), spéc. l'étude de K. Utz Tremp, « Die religiösen Laiengemeinschaften des Mittelalters (Beginen, Begarden), Kanton Freiburg, Stadt Freiburg ».

8. «Ortum conventus intravit in folio caulium albedinem quandam vidit et nulla benedictione premissa delectatione victus folium abscidit avideque comedit ac demonum quendam pessimum ignoranter deglutinivit. » Formicarius, III/1, p. 291 ; sur Heinrich Kalteisen, $c f$. TSCHACHER, Der Formicarius des Johannes Nider, p. 171-172.

9. «Nonnulle femine qui cito credunt et leves sunt corde satis putabant adesse Dei spiritum, ubi dyabolus locum habebat » Formicarius, III/1, p. 292.

10. «Et tamen a multis literatis tales pro fictis habebantur. » Formicarius, III/1, p. 292-293.

11. «Divinus amor (ut divinissimus docet Dionysius), non minores in quibusdam sed maiores habet effectus amore humano, quem constat rapi ad dilectum cogitationibus, et sese exprimere in gemitu, singultu, fletu, cantu et clamoribus. » Formicarius, III/1, p. 294-295.

12. Formicarius, II/4, p. 200 ; cf. Hansen, op. cit., p. 89-90; voir l'analyse détaillée de cette histoire par Catherine Chène dans L'Imaginaire du sabbat, p. 204-220; $c f$.

C. Ginzburg, op. cit. ; W. Tschacher, « Der Flug durch die Luft zwischen Illusionstheorie 
und Realitätsbeweis. Studien zum sog. Kanon Episkopi und zum Hexenflug ", Zeitschrift der Savigny-Stiftung zur Rechtsgeschichte, 116, Kan. Abt. 85, 1999, p. 225-276.

13. L'identification de ce juge avec Peter de Greyerz, longtemps acceptée, a été récemment mise en doute par Catherine Chène, L'Imaginaire du sabbat, p. 224-227.

14. L'Imaginaire du sabbat, p. 152-161.

15. L'Imaginaire du sabbat, p. 196-199.

16. Formicarius, V/9, p. 613.

17. Pour l'orientation générale, voir le début de l'article « extase » dans le Dictionnaire de Spiritualité Ascétique et Mystique (désormais abrégé DSAM), Paris, 1961, vol. IV, p. 2045-2189 ; I. M. LEWIS, Ecstatic Religion. An Anthropological Study of Spirit Possession and Shamanism, Harmondsworth, 1971. Pour une enquête récente sur les aspects médiévaux, voir N. CACIOLA, Discerning Spirits : Sanctity and Possession in the Later Middle Ages (Univ. of Michigan, Ph. D. thesis, 1994) ; EAD., « Wraiths, Revenants and Ritual in Medieval Culture », Past and Present, 152, 1996, p. 3-46 ; EAD., " Mystics, Demoniacs, and the Physiology of Spirit Possession in Medieval Europe ", Comparative Studies in Society and History, 42, 2000, p. 268-306 ; B. NewMAN, « Possessed by the Spirit : Devout Women, Demoniacs, and the Apostolic Life in the Thirteenth Century », Speculum, 73, 1998, p. 733-770.

18. M. Eliade, Le Chamanisme et les techniques archaïques de l'extase, Paris, 1951.

19. L. HonKo, "Role-taking of the shaman », Temenos, 4, 1969, p. 26-55 ; A.-L. SIIKALA, The Rite Technique of the Siberian Shaman, FF Communications 220, Helsinki, 1978, p. 330-341.

20. M. Eliade, op. cit. ; L. de Heusch, «Cultes de possession et religions initiatiques de salut en Afrique ", Annales du Centre d'Études des Religions, Bruxelles, 1962 ; S. M. Shirokogoroff, Psychomental Complex of the Tungus, Londres, 1935 ; I. M. Lewis, op. cit., p. 49-57.

21. C. Ginzburg, I Benandanti. Stregoneria e culti agrari tra Cinquecento e Seicento, Turin, 1966 ; trad. fr. : Les Batailles nocturnes, Paris, 1984.

22. C. Ginzburg, Le Sabbat, op. cit., n. 5.

23. M. Bos `ovic-Stulli, « Testimonianze orali croate e slovene sul Krsnik-Kresnik », Metodi e ricerche, N.S. 7, 1988, p. 32-50.

24. G. KLANICZAY, «Shamanistic elements in Central European Witchcraft », dans Shamanism in Eurasia, éd. par M. HoPPÁL, Göttingen, 1983, p. 404-422 ; une version amplifiée dans G. KLANICZAY, The Uses of Supernatural Power. The Transformation of Popular Religion in Medieval and Early Modern Europe, Cambridge, 1990, p. 129-150.

25. M. ELIADE, « Some Observations on European Witchcraft », History of Religions, 14, 1975, p. 149-172 ; G. KLIGMAN, Calus : Symbolic Transformation in Romanian Ritual, Chicago, 1981.

26. G. HeNNINGSEN, «The Ladies from Outside : an Archaic Pattern of the Witches' Sabbath », dans B. ANKARLoo et G. HENNINGSEN éd., Early Modern European Witchcraft : Centres and Peripheries, Oxford, 1990, p. 191-217.

27. É. Pócs, Between the Living and the Dead. A Perspective on Witches and Seers in the Early Modern Age, Budapest, 1998. Ead., "Le sabbat et les mythologies indo-européennes », dans N. Jacques-Chaquin et M. Préaud éd., Le Sabbat des sorciers XV ${ }^{e}-\mathrm{XVIII}^{e}$ s., Grenoble, 1993, p. 23-31.

28. P. DinZelBACHER, op. cit., p. 293. 
29. P. Brown, Le Culte des saints. Son essor et sa fonction dans la chrétienté latine, Paris, 1984 ; Id., "The Rise and Function of the Holy Man in Late Antiquity », dans Id., Society and the Holy in Late Antiquity, Berkeley/Los Angeles, 1982, p. 103-152, trad. fr. : La Société et le Sacré dans l'Antiquité tardive, Paris, 1985.

30. E. EVANS-PRITCHARD, Witchcraft, Oracles and Magic among the Azande, Oxford, 1937 ; P. BRown, « Sorcery, Demons and the Rise of Christianity from late Antiquity into the Middle Ages », dans M. Douglas éd., Witchcraft Confessions and Accusations, Londres, 1970, p. 17-45.

31. Pour ces accusations, voir A. A. BARB, « The Survival of Magic Arts ", dans

A. Momigliano éd., The Conflict between Paganism and Christianity in the Fourth Century, Oxford, 1963, p. 100-125.

32. Les sorcières sont décrites comme des «traitors within the gates » par P. Mayer, « Witches ", dans M. Marwick éd., Witchcraft and Sorcery. Selected Readings, Harmondsworth, 1970, p. 45-64.

33. Sur ces mouvements religieux, voir mon article « Religious Movements and Christian Culture. A Pattern of Centripetal and Centrifugal Orientations », dans G. Klaniczay, The Uses of Supernatural Power, op. cit., p. 28-50.

34. Historia miraculorum S. Bernardi in itinere germanico patratorum, PL, 185, col. 385-410 ; Epistula ad magistrum Archenfredum, ibid., col. 410-416 ; P.-A. Sigal, L'Homme et le miracle dans la France médiévale (XI ${ }^{e}-\mathrm{XII}^{e}$ s.), Paris, 1985, p. 18-20.

35. A. Vauchez, La Sainteté en Occident aux derniers siècles du Moyen Âge, Rome, 1981. 36. Ibid., p. 420-426. Je détaille davantage ce problème dans Klaniczay, The Uses of Supernatural Power, op. cit., p. 95-110.

37. M. Goodich souligne ces aspects utilitaires et en même temps communautaires de la sainteté de la fin du Moyen Âge dans Violence and Miracle in the Fourteenth Century. Private Grief and Public Salvation, Chicago/Londres, 1995.

38. G. Zarri, Le sante vive. Profezie di corte e devozione femminile tra ' 400 e '500, Turin, 1990 ; P. Dinzelbacher, op. cit., p. 119-125.

39. On trouvera davantage sur les sources concernant ces saints dans A. Vauchez, op. cit. ; R. Bell, Holy Anorexia, Chicago/Londres, 1985 ; C. W. Bynum, Holy Feast and Holy Fast. The Religious Significance of Food to Medieval Women, Berkeley/Los Angeles, 1987, trad. fr. : Jeûnes et festins sacrés. Les femmes et la nourriture dans la spiritualité médiévale, Paris, 1994 ; voir aussi mon « Modelli di santità femminile tra i secoli xiii e xiv in Europa Centrale e in Italia ", dans Spiritualità e lettere nella cultura ungherese del basso medioevo, éd. S. Graciotti et C. Vasoli, Florence, 1995, p. 75-110.

40. Formicarius, IV/9, p. 483 ; Raymundus de Capua, Vita Sanctae Catharinae Senensis. Legenda maior. AA SS Aprilis Tom III, Venezia 1738, coll. 584-959.

41. Formicarius, IV/9, p. 484 ; P. Dinzelbacher, « Colet(t)a », Lexikon des Mittelalters, Munich, 1984, vol. III/1, p. 30 ; Pierre de Vaux, Vie de Sœur Colette, introduction, transcription et notes par E. Lopez, Saint-Étienne, 1994 ; E. Lopez, Culture et sainteté. Colette de Corbie (1381-1447), Saint-Étienne, 1994.

42. Formicarius, IV/9, p. 486.

43. Ibid., II/1, p. 163.

44. Ibid., II/2, p. 183-184.

45. Ibid., III/11, p. 391-393 : « Ostenderat autem se predicta femina saepe numero jacere, velut in ecstasi \& in raptu ecstatico. Ex quo reversa \& expergiscens, suis postmodum secreta, quae non noverat, dicere solebat... certa die, quae nominabatur, 
quinque Christi stigmatum insignia, in manibus, pedibus, \& in corde feminae certitudinaliter apparerent $»$.

46. Ibid., III/8, p. 361-365. Voir P. DinZelbacheR, op. cit., p. 91-93 ; P. DinZELBACHER et K. RuH, « Magdalena von Freiburg », dans Die deutsche Literatur des Mittelalters.

Verfasserlexikon, vol. IV, p. 1117-1121.

47. Formicarius, III/8, p. 362-363.

48. A. M. KLEINBERG, Prophets in Their Own Country. Living Saints and the Making of Sainthood in the Later Middle Ages, Chicago, 1992.

49. J. von GöRRES, Die christliche Mystik, Ratisbonne, 1836, vol. III, p. 668 ; H. THURSTON, The Physical Phenomena of Mysticism, Londres, 1952.

50. P. Debognie, "Essai critique sur l'histoire des stigmatisations au Moyen Âge ", Études Carmélitaines, 21.2, 1936, p. 22-59 ; J. M. Höcht, Von Franziskus zu Pater Pio und Therese Neumann. Eine Geschichte der Stigmatisierten, Aschaffenburg/Stein am Rhein, 1974 ; A. Vauchez, « Les stigmates de saint François et leurs détracteurs dans les derniers siècles du Moyen Âge ", Mélanges de l'École française de Rome, 80, 1968, p. 595-625 ; C. Frugoni, Francesco e l'invenzione delle stimmate. Una storia per parole e immagini fino a Bonaventura e Giotto, Turin, 1993 ; A. DAvIDSON, « Miracles of Bodily Transformation, or, How St. Francis Received the Stigmata », dans C. A. JonES, P. Gallison, A. Slaton éd., Picturing Science, Producing Art, Londres, 1998, p. 101-124. 51. P. OCHSENBEIN, « Leidensmystik in dominikanischen Frauenklöstern des 14. Jahrhunderts am Beispiel der Elsbeth von Oye ", dans P. DinZELBACHER, D. R. BAUER éd., Religiöse Frauenbewegung und mystische Frömmigkeit im Mittelalter, Cologne/Vienne, 1988, p. 353-372, sp. 361-366.

52. Hugo de SANCTO Victore, In hierarchiam coelestem, 3, 2, PL 175, col. 983c. DS, col. 2113 ; pour Nider, cf. Formicarius, III/1, p. 294.

53. F. Vanderbrouke, « Discernement des esprits », DSAM, vol. III, p. 1254-1266; G. SWITEK, «Discretio spirituum. Ein Beitrag zur Geschichte der Spiritualität », Theologie und Philosophie, 47, 1972, p. 36-76.

54. A. ZUMKeller et R. G. WARNock, Der Traktat Heinrichs von Friemar über die Unterscheidung der Geister, Würzburg, 1977 (Cassiciacum, 32).

55. T. HOHMANN, Heinrichs von Langenstein "Unterscheidung der Geister » lateinisch und deutsch. Texte und Untersuchungen zu Übersetzungsliteratur aus der Wiener Schule, Zurich/ Munich, 1977 ; A. MORISI GUERRA, « Il silenzio di Dio e la voce dell'anima. Da Enrico di Langenstein a Gerson ", Cristianesimo nella storia, 17, 1996, p. 393-413.

56. P. Boland, The Concept of Discretio Spirituum in John Gerson's « De Probatione Spirituum » and « De Distinctione Verarum Visionum a Falsis », Washington D.C., 1959 ; Entre Dieu et Satan. Les visions d'Ermine de Reims (†1396), prés., éd. et trad. par C. Arnaud-Gillet, Florence, 1997 ; B. P. McGuire, « Late medieval care and control of women : Jean Gerson and his sisters ", Revue d'histoire ecclésiastique, 92, 1997, p. 5-37.

57. S. von HEUSINGER, Johannes Mulberg OP († 1414). Ein Leben im Spannungsfeld von Dominikanerobservanz und Beginenstreit, Berlin, 2001.

58. W. Williams-KraPP, loc. cit.

59. A. Vauchez, « La naissance du soupçon : vraie et fausse sainteté aux derniers siècles du Moyen Âge ", dans Id., Saints, prophètes et visionnaires. Le pouvoir surnaturel au Moyen Âge, Paris, 1999, p. 208-219. Id., «L'Église face au mysticisme et au prophétisme aux derniers siècles du Moyen Âge ", dans Id., Les Laïcs au Moyen Âge. Pratiques et expériences religieuses, Paris, 1987, p. 259-265. 
60. Formicarius, V/8, p. 600-602 : «fassa est, se habere familiarem Dei angelum, qui judicio literatissimorum virorum judicatus est esse malignus spiritus ex multis conjecturis \& probationibus, per quem spiritum velut magam effectam ignibus per publicam iustitiam consumi permiserunt. »

61. Sur ce point, on peut voir É. Anheim, J.-P. Boudet, F. Mercier, M. Ostorero, « Aux sources du sabbat. Lectures croisées de L'Imaginaire du sabbat. Édition critique des textes les plus anciens (1430 ca-1440 ca) », Médiévales, 42, printemps 2002, p. 153-175.

62. J. HANSEN, «Inquisition und Hexenverfolgung im Mittelalter », Historische Zeitschrift, 81, 1898, p. 385-432, en particulier p. 386.

63. Plus récemment, voir N. Cohn, Europe's Inner Demons. An Enquiry Inspired by the Great Witch-Hunt, New York, 1975.

64. Comme en 1459, à Arras pendant une des plus terribles persécutions, voir J. Hansen, op. cit. n. 4, p. 467, 476, 556, 569. F. Mercier, L'Enfer du décor. La Vauderie d'Arras (1459-1491) ou l'émergence contrariée d'une nouvelle souveraineté autour des ducs Valois de Bourgogne ( $\mathrm{XV} v^{e} \mathrm{~s}$ ), thèse dactylographiée, université Lyon II-Lumière, décembre 2001.

65. R. I. Moore, La Persécution : sa formation en Europe, tr. fr., Paris, 1991 ; H. Zaremska, Les Bannis au Moyen Âge, Paris, 1996 ; D. Nirenberg, Communities of Violence: Persecution of Minorities in the Middle Ages, Princeton, 1996, trad. fr. : Violence et minorités au Moyen Âge, Paris, 2001. Voir le lien avec les stéréotypes de la sorcellerie chez C. Ginzburg, Le Sabbat, op. cit., chap. I.

66. J. Marx, L'Inquisition en Dauphiné. Étude sur le développement et la répression de l'hérésie et de la sorcellerie du XIV ${ }^{e}$ s. au début du règne de François $I^{e r}$, Paris, 1914 ; plus récemment G. G. Merlo, Eretici e inquisitori nella società piemontese del Trecento, Turin, 1977 ; A. Blauert, Frühe Hexenverfolgungen : Ketzer-, Zauberei- und Hexenprozesse des 15. Jahrhunderts, Hambourg, 1989 ; B. Andematten et K. Utz Tremp, « De l'hérésie à la sorcellerie : l'inquisiteur Ulric de Torrenté OP (vers 1420-1445) et l'affermissement de l'inquisition en Suisse romande ", Revue d'histoire ecclésiastique suisse, 86, 1992, p. 69-119 ; P. Paravy, De la chrétienté romaine à la réforme en Dauphiné. Évêques, fidèles et déviants (vers 1340-1530), Rome, 1993, I-II ; et différents volumes des Cahiers Lausannois d'Histoire Médiévale, édités par A. Paravicini Bagliani : C. Chène, Juger les vers. Exorcismes et procès d'animaux dans le diocèse de Lausanne ( $\mathrm{XV}^{e}-\mathrm{XVI}{ }^{e} \mathrm{~S}$.), Lausanne, 1995 ; M. Ostorero, "Folâtrer avec les démons ». Sabbat et chasse aux sorciers à Vevey (1448), Lausanne, 1995 ; E. Maier, Trente Ans avec le diable. Une nouvelle chasse aux sorciers sur la Riviera lémanique (1477-1484), Lausanne, 1996 ; S. Strobino, Françoise sauvée des flammes? Une Valaisanne accusée de sorcellerie au XV s., Lausanne, 1996.

67. R. Kieckhefer, Magic in the Middle Ages, Cambridge, 1989, p. 151-200 ; Id., " The Specific Rationality of Medieval Magic ", American Historical Review, 99, 1994, p. 833-836 ; plus récemment, M. Bailey, loc. cit. n. 5.

68. L'Imaginaire du sabbat, p. 152-161.

69. K. Schieler, op. cit., n. 1, p. 269-341.

70. Formicarius III/10, p. 386.

71. A. Blauert, op. cit., p. 36-50 ; B. Andenmatten et K. Utz Tremp, loc. cit.

72. Formicarius, V/5, p. 560 ; L'Imaginaire du sabbat, p. 152.

73. Formicarius, V/4, p. 551-552; L'Imaginaire du sabbat, p. 164-166; W. Tschacher, op. cit., n. 1, p. 173, 401-402.

74. Formicarius, V/5, p. 567-569.

75. Ibid., I/2, p. 65-66. 
76. Ibid., V/4, p. 548 ; L'Imaginaire du sabbat, p. 158-164.

77. Ibid., V/3, p. 544 et V/4, p. 554 et 559 ; L'Imaginaire du sabbat, p. 152, 170.

78. L'Imaginaire du sabbat, p. 355-437.

79. Une partie de ces procès est éditée par J. Hansen, op. cit., p. 539-544; analysée par J. Marx, op. cit., p. 32-43 ; P. Paravy, op. cit., p. 783 et sq., avec une évaluation statistique : entre 1424 et 1446, sur 258 accusés (83 hommes et 175 femmes), 151 furent condamnés à mort.

80. Hansen, op. cit., p. 118-122 ; nouvelle édition par M. Ostorero et K. Utz Tremp dans L'Imaginaire du sabbat, p. 267-337.

81. Hansen, op. cit., p. 533-537; nouvelle édition par K. Utz Tremp dans L'Imaginaire du sabbat, p. 23-61.

82. P. Paravy, «À propos de la genèse médiévale des chasses aux sorcières : le traité de Claude Tholosan, juge dauphinois (vers 1436) », Mélanges de l'École française de Rome, 91, 1979, p. 333-379 ; A. Blauert, op. cit., p. 50-70 ; C. Ginzburg, op. cit. ; M. Bailey, loc. cit. ; les commentaires dans L'Imaginaire du sabbat ; M. Ostorero, " The Concept of the Witches' Sabbath in the Alpine Region (1430-1440). Text and Context », à paraître dans G. Klaniczay et É. Pócs éd., Demons, Spirits and Witches, CEU Press, Budapest.

83. J'emprunte le terme du livre de R. Muchembled, La Sorcière au village (XVe-XVIII ${ }^{e} \mathrm{~s}$ ), Paris, 1979.

84. P. Paravy, loc. cit., p. 356-357 ; L'Imaginaire du sabbat, p. 364-367.

85. Ce motif apparaît dans un procès mené par Tholosan : J. Marx, op. cit., p. 36-39.

86. J. Hansen, op. cit., p. 535 ; L'Imaginaire du sabbat, p. 36.

87. R. Jakobson et M. Szeftel, « The Vseslav Epos«, dans R. Jakobson, Selected Writings. IV. Slavic Epic Studies, La Haye-Paris, 1966, p. 301-379 ; L. Harf-Lancner, « La métamorphose illusoire : des théories chrétiennes de la métamorphose aux images médiévales du loup-garou ", Annales ESC, 40, 1985, p. 208-226 ; C. Ginzburg, op. cit.

88. J. Hansen, op. cit., p. 120 ; L'Imaginaire du sabbat, p. 292.

89. J. Hansen, op. cit., p. 120 ; L'Imaginaire du sabbat, p. 294.

90. J. Hansen, op. cit., p. 121 ; L'Imaginaire du sabbat, p. 296.

91. G. Klaniczay, "Hungary : The Accusations and the Universe of Popular Magic », dans B. Ankarloo et G. Henningsen éd., Early Modern European Witchcraft : Centres and Peripheries, Oxford, 1990, p. 250-251 ; ID., « Le sabbat raconté par les témoins des procès de sorcellerie en Hongrie », dans N. Jacques-Chaquin et M. Préaud éd., op. cit., n. 9, p. 232-234.

92. J. Hansen, op. cit., p. 534 ; L'Imaginaire du sabbat, p. 32.

93. J. MARX, op. cit., p. 39.

94. A. BORST, loc. cit.

95. A. MACFARLANE, Witchcraft in Tudor and Stuart England. A Regional and Comparative Study, New York/Evanston, 1970 ; K. THOMAS, Religion and the Decline of Magic. Studies in popular beliefs in sixteenth and seventeenth century England, Londres, 1971 ; J. P. DEMOS, Entertaining Satan. Witchcraft and the Culture of Early New England, Oxford, 1982.

96. Par exemple : le Christ et Satan apparaissent sous la forme humaine ; l'érotisme est impliqué dans l'amour ressenti pour l'un comme pour l'autre : étreindre, toucher, embrasser (les stigmates, les lèvres, le derrière) ; l'union physique avec le Fiancé Céleste dans l'extase, et les plaisirs liés à l'orgasme ; les détails obscènes de copulation avec le démon ; les grossesses sataniques ou célestes ; les témoignages de fiançailles célestes comparées aux fiançailles avec Satan ; les anneaux, les stigmates, la marque de la sorcière, les fêtes démoniaques ou célestes, les danses. Voir J. LEUBA, The Psychology of 
Religious Mysticism, New York-Londres, 1925, p. 137-157 ; C. W. BYNUM, op. cit. et EAD., Fragmentation and Redemption. Essays on Gender and the Human Body in Medieval Religion, New York, 1991. Les parallèles entre ces deux mythologies ont été analysés récemment par M. Craveri, Sante e streghe, Milan, 1980 ; R. Bell, op. cit. ; G. Zarri, loc. cit. ; P. Dinzelbacher, op. cit. : en rapport avec ce dernier, un certain nombre d'objections ont été faites par R. Kieckhefer, op. cit., p. 368-373 ; j'ai moi-même attiré l'attention sur cette même opposition binaire entre les saints et les sorcières dans « Miraculum und Maleficium. Einige Überlegungen zu den weiblichen Heiligen des Mittelalters in Mitteleuropa ", Wissenschaftskolleg Jahrbuch, 1990/91, p. 224-252 ; et « Miraculum and Maleficium : Reflections Concerning Late Medieval Female Sainthood », dans Problems in the Historical Anthropology of Early Modern Europe, R. Po-Chia Hsia et R. W. Scribner éd., Wiesbaden, 1997, p. 49-74.

97. F. Bonney, «Autour de Jean Gerson. Opinions des théologiens sur les superstitions et la sorcellerie au début du xv $\mathrm{x}^{\mathrm{e}}$ s. ", Le Moyen Âge, 77, 1971, p. 85-98. J.-P Boudet, « Les condamnations de la magie à Paris en 1398 ", Revue Mabillon, nouvelle série, 12 (t. 73), 2001, p. 121-157, et J. Véronèse, « Jean sans Peur et la "fole secte" des devins : enjeux et circonstances de la rédaction du traité Contre les devineurs (1411) de Laurent Pinon », Médiévales 40, printemps 2001, p. 113-132.

98. J. B. RUSSELL, Witchcraft in the Middle Ages, Ithaca/Londres, 1972.

99. Dorothée de Montau guérit des maléfices, voir R. STACHNIK, Die Akten des Kanonisationsprozesses Dorotheas von Montau von 1394 bis 1521, Cologne/Vienne, 1978, p. 108-109 et 473-474, et R. KIECKHEFER, loc. cit., p. 359.

100. J.-M. Sallmann, Chercheurs de trésors, jeteurs de sorts. La quête du surnaturel à Naples au XVI ${ }^{e}$ s., Paris, 1986. Id., Naples et ses saints à l'âge baroque (1540-1750), Paris, 1994.

101. I. Collijn éd., Acta et processus canonizationis beatae Birgitte, Uppsala, 1924-31, p. 513 ; M. Goodich, op. cit., p. 64-66.

102. Т. DöмÖтӧR, « The Cunning Folk in English and Hungarian Witch Trials », dans V. NEWALl éd., Folklore Studies in the Twentieth Century. Proceedings of the Centenary Conference of the Folklore Society, Woodbridge-Totowa, 1980, p. 183-187 ; W. DE BLÉCOURT, « Witch Doctors, Soothsayers and Priests. On Cunning Folk in European Historiography and Tradition ", Social History, 19, 1994, p. 285-303.

\section{RÉSUMÉS}

Achevé aux alentours de 1435, le Formicarius de Johannes Nider (1380-1438) décrit des saints et des hérétiques, des visions, des révélations, des phénomènes de possession et de simulation, des vertus extraordinaires et des péchés mortels, des opérations miraculeuses, et des œuvres de magiciens et de sorciers. Pour expliquer la signification extraordinaire de la transe religieuse dans ces histoires, nous proposons d'examiner l'hypothèse d'une corrélation entre le chamanisme, les manifestations d'extase religieuse et l'émergence des croyances en la sorcellerie diabolique. Suivant le schéma conceptuel fort original de Nider qui juxtapose les visions d'origine céleste ou diabolique, nous étudions successivement les liens du chamanisme avec la sainteté des visionnaires de la fin du Moyen Âge, puis avec le sabbat des sorcières, pour finir par montrer 
dans quelle mesure la transe peut être considérée comme un point commun entre la sainte et la sorcière, qui constituent un système binaire dont la polarité bascule au $\mathrm{xv}^{\mathrm{e}}$ siècle.

Between angelic visions and shamanistic trance: the Witches' Sabbath in the Formicarius of Nider. The Formicarius of Johannes Nider (1380-1438), finished around 1435, describes saints and heretics, visions, revelations, phenomena of possession and simulation, extraordinary virtues and mortal sins, miraculous workings and the deeds of magicians and sorcerers. To explain the extraordinary significance of religious trance in these stories, we propose to examine the hypothesis of a correlation between shamanism, religious ecstasy and the emergence of the beliefs in diabolic witchcraft. Following the very original conceptual scheme of Nider, who juxtaposes visions of heavenly and diabolic origin, we analyse successively the relationship between shamanism and visionary sainthood at the end of the Middle Ages, then between the former and the witches' sabbath, to able to show, finally, in what ways could the trance be considered as a common point between the saint and the witch, who constitute a binary system with a shaky polarity in the 15 th century.

\section{INDEX}

Mots-clés : sorcellerie, chamanisme, sainteté, Johannes Nider, Formicarius

Keywords : witchcraft, shamanism, sainthood

\section{AUTEUR}

\section{GÁBOR KLANICZAY}

Collegium Budapest, 1014 Budapest, Szentháromság u. 2. Hongrie 